\title{
La visión de la educación ambiental de estudiantes de maestría en pedagogía en el marco de la Cátedra de la Paz en Colombia
}

\author{
Carmen Solís-Espallargas ${ }^{(1) *}$ y Carlos H. Barreto-Tovar(2) \\ (1) Facultad de Ciencias de la Educación, Dpto. de Didáctica de las Ciencias Experimentales y Sociales, Universidad de \\ Sevilla, C/ Pirotecnia s/n, Sevilla-España. (correo-e: carmensolise@us.es) \\ (2) Facultad de Educación, Universidad de La Sabana, Campus del Puente del Común, Km. 7, Autopista Norte de \\ Bogotá. Chía, Cundinamarca-Colombia. (correo-e: carlos.barreto2@unisabana.edu.co) \\ * Autor a quien debe ser dirigida la correspondencia.
}

Recibido Oct. 8, 2019; Aceptado Nov. 29, 2019; Versión final Dic. 24, 2019, Publicado Abr. 2020

\begin{abstract}
Resumen
Se analizan las visiones de estudiantes de posgrado sobre los problemas del mundo, la sostenibilidad y sus propuestas sobre educación ambiental contextualizados en el marco de la Cátedra de la Paz en Colombia. Mediante un cuestionario participaron en esta investigación 175 estudiantes de Maestría en Pedagogía de la Universidad de la Sabana (Colombia). Los resultados muestran la violencia y la contaminación ambiental como los problemas más destacados. El concepto de sostenibilidad lo identifican principalmente con toma de conciencia. Ellos también entienden que entre los fines de la educación ambiental debe estar el conocimiento de la naturaleza, desde una implicación emocional de cuidado y protección, pero que lleve a la acción para el cambio. Las conclusiones nos ofrecen orientaciones para la mejora de la formación de los estudiantes de posgrado, entre ellas el tratamiento de la problemática socioambiental desde un enfoque holístico y complejo y la inclusión de herramientas para la educación para la paz y resolución de conflictos socioambientales.
\end{abstract}

Palabras clave: educación ambiental; formación docente; sostenibilidad; educación para la paz; posconflicto

\section{The environmental education vision of master's in pedagogy students in the framework of the Cátedra de la Paz in Colombia}

\begin{abstract}
The aim of this research is analysing the visions of master's students on global problems, sustainability and their suggestions for Environmental Education within the framework of the Cátedra de la Paz in Colombia. Students of the Master's Degree in Pedagogy of the Universidad de la Sabana (Colombia) participated in a questionnaire. Results showed that violence and environmental pollution were considered the most relevant problems. The concept of sustainability was mainly referred to as environmental awareness. Participants also understand that Environmental Education ought to include knowledge about nature. Students should be emotionally involved in the care and protection of the environment and future strategies need to be suggested Teachers' training needs to be improved regarding social and environmental problems. A complex and holistic perspective should be applied, and teacher training programs should provide students with the necessary tools and strategies for including peace and the environment in their learning.
\end{abstract}

Keywords: environmental education; teacher training; sustainability; peace education; post-conflict 


\section{INTRODUCCIÓN}

La Agenda 2030 (United Nations, 2015) contempla 17 Objetivos de Desarrollo Sostenible (ODS) que abordan los principales retos y desafíos en los que la humanidad debe progresar para reconducir el desarrollo hacia un modelo más sostenible. Los objetivos están formulados de manera que no es posible construir un mundo pacífico si no se adoptan medidas para un desarrollo económico y social de todas las personas en todos los lugares y garantizar la protección de los derechos humanos. Los ODS afrontan grandes desafíos como la crisis climática, la pobreza, el hambre, la salud, la educación, la igualdad de género, el agua, el saneamiento, la energía, el medio ambiente y la justicia social (Rieckmann, 2017). Uno de los requisitos para el logro de los ODS se basa en la construcción de sociedades pacíficas, justas e inclusivas tal y como se recoge en el ODS 16: paz, justicia e instituciones fuertes.

La educación es uno de los elementos clave para el logro de conseguir la implementación de la sostenibilidad en la vida cotidiana. En el campo de la educación para la paz no es nueva la tarea de vincularla con la educación social, ambiental y económica en un enfoque integral (Brantmeier, 2013). La educación ambiental (en adelante EA) y la educación para la paz mantienen interconexiones claves, complementariedades y sinergias en la construcción de un mundo que sea justo y sostenible y no violento de forma que se respete los derechos de toda forma de vida. Ambas persiguen el desarrollo de una educación crítica que permita una transformación personal para la transformación del sistema. Además comparten propósitos comunes basados en la comprensión de las causas de los conflictos y el desarrollo de estrategias adecuadas para la construcción de una paz ante el afrontamiento de los problemas ambientales (Swee-Hin, y Cawagas, 2010). En esta línea la investigadora pionera de la paz Betty Reardon argumentó que la Educación para la paz debería inculcar los valores de responsabilidad ambiental, diversidad cultural, solidaridad humana, responsabilidad social e igualdad de género (Reardon, 1999).

Numerosas investigaciones vienen alertando de cómo la crisis climática y la violencia contribuyen a la destrucción, el sufrimiento y la muerte. En muchos casos de catástrofe, el daño ambiental puede ser irreversible. En esta línea Naciones Unidas está alertando sobre las consecuencias del cambio climático mediante la campaña Acción climática por la paz (2019). Esta campaña está centrada en combatir el cambio climático con el fin de proteger y promover la paz en todo el mundo. En palabras del Secretario General de la ONU, António Guterres: "Hoy la paz se enfrenta a un nuevo peligro: la emergencia climática que amenaza nuestra seguridad, nuestro sustento y nuestras vidas" (citado en https://www.un.org/es/globalcategories/peace-and-security). Y es que las consecuencias de la crisis climática como son los refugiados ambientales desplazados por desastres naturales, la salinización del agua y los cultivos o las tensiones sobre los recursos naturales están provocando conflictos en la mayoría de países. De este modo, el desarrollo sostenible se convierte como uno de los contenidos de aprendizaje clave junto con la cultura de la paz que deben estar presentes de forma transversal en las instituciones educativas.

Concretamente en Colombia, las problemáticas sociales por conflicto armado que han acontecido por más de seis décadas, han desencadenado una serie de conflictos ambientales -dentro de los que se incluyen también los sociales- que han llevado a re-pensar la finalidad y formas de educación desde diferentes ámbitos, buscando alcanzar un desarrollo integral y que propenda por la formación de país. De este modo, se ha apostado por la formación para el desarrollo sostenible y la paz, a través de los Proyectos Escolares Ambientales PRAE (Decreto 1743 de 1994 y Acuerdo 407 de 2015) y la Cátedra para la Paz (Ley 1732 de 2014 y Decreto 1038 de 2015). Los PRAE son una apuesta común entre los Ministerios del Medio Ambiente y el Ministerio de Educación Nacional, donde se apuesta por la formación de una ciudadanía responsable desde una visión sistémica del ambiente que considera las principales problemáticas contextuales y busca las oportunas soluciones a las mismas. Por su parte, la Cátedra de la Paz, se presenta como un eje articulador de la educación ambiental en las escuelas que ayude a orientar los pasos para la construcción de ciudadanía desde "el proceso de apropiación de conocimientos y competencias relacionados con el territorio, la cultura, el contexto económico y social y la memoria histórica [...]" (art. 2, Decreto 1038 de 2015). Ambas iniciativas se enmarcan dentro de la educación formal básica y media de todo el país a partir de la propuesta de transversalización con todas las áreas de conocimiento y además, demandan de una pertinente formación de los profesores que permita desarrollar un pensamiento sistémico y holístico a partir de la generación de estrategias que garanticen la responsabilidad individual y colectiva, la solidaridad y la promoción de principios éticos (Cortés y González, 2017).

Si los futuros educadores van a reemplazar el pensamiento con una visión integrada en la planificación para el logro de una cultura de paz social y ecológica, es tarea de los docentes universitarios prepararlos para ello. Los trabajos de Cebrian y Juyent (2015) y Varela-Losada et al. (2014), concluyen que son muchos los estudiantes de educación que reconocen la necesidad de mejorar sus conocimientos y herramientas en sostenibilidad. Shapiro (2015) destaca el potencial de humanizar la educación, primordialmente la universitaria, para promover sociedades equitativas y justas, especialmente después del conflicto. En la 
misma línea Nussbaum (1996) sugiere que la educación, debe enfatizar la responsabilidad hacia la humanidad y los valores compartidos como base para trascender las desigualdades e injusticias a nivel global, nacional y local y, por lo tanto, para construir y mantener una paz sostenible. Con este fin, los formadores universitarios deben considerar estrategias pedagógicas que integren las demandas sociales y ecológicas del presente y el futuro de manera equilibrada (Verhagen, 2014).

Como ejemplo de investigaciones de este campo en países de conflicto y posconflicto, destacamos los trabajos de formación de profesorado en EA para el desarrollo sostenible en UZNA Universidad de Zambia (Namafe, 2015) en una situación política de anocracia (una anocracia no es ni completamente democrática ni completamente autocrática, y puede estar en transición de una a la otra, World Bank, 2008); los trabajos de Tal y Peled (2017) sobre el mapeo de la situación de la EA en las escuelas de Israel y las dificultades encontradas en pro de una mejora de la formación docente; en Kosovo se investigó cómo los maestros abordan los problemas ambientales relevantes a nivel local en el aula desde los enfoques de enseñanza relacionados con la educación para el desarrollo sostenible concluyendo cómo un programa formativo de capacitación docente para la mejora de la educación ambiental contribuye a una educación ambiental más eficaz (Spahiu y Lindemann-Matthies, 2015); y en Pakistán se investigó sobre un estudio de conciencia ambiental de los maestros en formación en comparación con los estudiantes de magisterio suecos concluyendo la necesidad de reformular los programas de formación de maestros para incidir en una mejora de la formación en educación ambiental (Kalsoom et al., 2017).

Lara (1996), citado por Duarte y Valbuena (2014), plantea que para Colombia (en el marco del proyecto auspiciado por la OEI sobre Educación Ambiental en Iberoamérica) tras la Reunión Técnica celebrada en 1996 en Villa de Leyva, se realizó una descripción de las características de formación en educación ambiental, planteando criterios y estrategias metodológicas y proponiendo un perfil de los educadores ambientales. La reunión concluyó en recomendaciones que describen lo necesario para encontrar estrategias de formación que permitan a los educadores construir su propio discurso pedagógico". Desde entonces Duarte y Valbuena (2014) destacan a partir de una revisión en las publicaciones especializadas en educación ambiental, la necesidad de continuar con trabajos de investigación y de evaluación de experiencias que tengan como objeto la mejora de la formación de estudiantes de educación en este campo.

Es por ello que se realiza este trabajo con la finalidad de ofrecer los conocimientos y visiones sobre sostenibilidad y educación ambiental para una cultura de paz que tienen los maestros en formación de posgrado con el fin de incorporarlo en las mejoras de propuestas de programas de formación docente. Partimos de la premisa que para que los maestros puedan tratar de forma significativa la problemática social y ambiental en sus escuelas resulta imprescindible un conocimiento profesional del maestro que integre por un lado el conocimiento académico que reside en la formación y adquisición de conocimientos socioambientales y pedagógicos; por otro lado el conocimiento que se adquiere de la experiencia y que ayuda a desarrollar competencias relacionadas con el saber hacer; y por último con la propia cosmovisión del maestro que reside en el compromiso ético con los problemas sociales y la sostenibilidad global y ambiental (García, 2004). Concretamente en este trabajo nos hemos centrado en investigar parte de la cosmovisión que tienen los maestros y maestras en formación de posgrado sobre la problemática del mundo, cuáles son sus visiones sobre sostenibilidad y las finalidades de la educación ambiental, enfoques que proceden de la propia historia personal y percepción del mundo. El grado de conocimiento, importancia e implicación que tengan los maestros sobre la sostenibilidad y la educación ambiental resultará crucial para la mejora de los programas de Maestría en Pedagogía que atienda sus visiones y necesidades en un contexto socioambiental de posconflicto.

\section{METODOLOGIA}

La muestra participante en esta investigación está formada por 175 estudiantes de la Maestría en Pedagogía de la Universidad de la Sabana (Colombia) que cuentan con la particularidad de que simultánea-mente a los estudios de posgrado están ejerciendo como maestros y maestras. Es precisamente esta particularidad la que suscita el interés de los investigadores por llevar a cabo este trabajo con ellas y ellos y de realizar los análisis de datos y conclusiones teniendo en cuenta estas dos características (como estudiantes de posgrados y maestros en ejercicio). Los estudiantes realizan su actividad profesional como maestros en educación inicial, básica y media de Bogotá y Cundinamarca (centro de Colombia), en colegios estatales de nivel socioeconómico medio y bajo. La muestra está formada por un $75 \%$ de mujeres y un $25 \%$ de hombres con edades comprendidas entre los 27 y 50 años. Son becarios beneficiados de la Secretaría de Educación de Bogotá o del Ministerio de Educación Nacional. La mayoría de los participantes tienen un nivel de formación de licenciados en educación y profesionales que no tienen formación inicial en educación.

Con el fin último de mejorar la formación en educación ambiental para la paz de estos estudiantes de la Maestría en Pedagogía (maestros en ejercicio), este trabajo tiene los siguientes objetivos: 1) Identificar las 
visiones que tienen los estudiantes de la Maestría sobre los problemas del mundo, en qué medida aparecen problemáticas ambientales y sociales y qué prioridad por orden de importancia les asignan a los problemas; 2) conocer cuáles son las propuestas que realizan los estudiantes de la Maestría para la minimización y/o resolución de las problemáticas que declaran; 3) analizar las principales concepciones que tienen los estudiantes de la Maestría sobre el concepto de sostenibilidad; y 4) Indagar en las visiones de estudiantes de la Maestría en cuanto a las finalidades y enfoques de la educación ambiental en el contexto escolar.

La recogida de datos se realizó mediante un cuestionario elaborado a partir del resultado de acciones formativas y trabajos anteriores (Solís-Espallargas y Valderrama-Hernández, 2015). Está estructurado en base a dos tipos de preguntas: preguntas abiertas y preguntas de elección múltiple tipo Likert previamente validado mediante expertos y con una submuestra para comprobar que se ajusta a los objetivos establecidos. La escala Likert presenta un valor de $\alpha$ de Cronbach de 0,8 considerado suficiente (Oviedo y Campo-Arias, 2005). La primera parte del cuestionario está compuesta por tres preguntas abiertas que corresponden con los objetivos 1, 2 y 3 de esta investigación. En la segunda parte que corresponde con el objetivo 4 se explicitan las instrucciones necesarias para rellenar datos tipo Likert.

El análisis y organización de la información se desarrolló acorde al tipo de pregunta. Para la primera parte formada por preguntas abiertas se realizó el siguiente procedimiento: a) vaciado de la información y organización de los datos, b) negociación y formulación de las categorías de análisis (inicial), c) aplicación de las categorías emergentes a los datos, d) triangulación de los datos y e) cierre del sistema de categorías mediante triangulación con un $100 \%$ de acuerdo entre los investigadores. El análisis de la segunda parte del cuestionario tipo Likert se procedió con un vaciado de los datos y su organización mediante la estadística descriptiva expresada en frecuencias y porcentajes.

\section{RESULTADOS Y DISCUSIÓN}

Los resultados se presentan por separado para cada parte del cuestionario. Una primera parte del cuestionario abordando las preguntas 1 a 3 y una segunda parte donde se recogen los los datos del cuestionario tipo Likert.

\section{Primera parte del cuestionario.}

\section{Pregunta 1:}

En los medios de comunicación hay continuas referencias sobre los problemas del mundo; de ellos indica los 5 problemas que te preocupan más, ordénalos de mayor a menor importancia para ti. Para el análisis nos hemos valido de un sistema de categorías emergente como consecuencia de un proceso de codificación inductiva, siendo el sistema de categorías el esquema vertebrador de los conceptos presentes en la información analizada (ver Tabla 1).

Tabla 1: Sistema de categorías y subcategorías

\begin{tabular}{|c|c|c|c|c|c|c|c|}
\hline Categoría & Subcategoría & Categoría & Subcategoría & Categoría & Subcategoría & Categoría & Subcategoría \\
\hline \multirow{6}{*}{$\begin{array}{l}\text { 1. Problemas } \\
\text { Ambientales }\end{array}$} & $\begin{array}{l}\text { 1.1. Cambio } \\
\text { climático }\end{array}$ & \multirow{11}{*}{$\begin{array}{l}\text { 3. Problemas } \\
\text { Sociales }\end{array}$} & $\begin{array}{l}\text { 3.1. Crisis de } \\
\text { valores }\end{array}$ & \multirow{3}{*}{ 4. Poder } & $\begin{array}{l}\text { 4.1. Abuso de } \\
\text { poder }\end{array}$ & \multirow{9}{*}{ 9. Otras } & $\begin{array}{l}\text { 9.1. Contratos } \\
\text { sociales }\end{array}$ \\
\hline & $\begin{array}{l}1.2 . \\
\text { Contaminación } \\
\text { ambiental }\end{array}$ & & 3.2. Pobreza & & 4.2. Corrupción & & $\begin{array}{l}\text { 9.2. Falta de } \\
\text { cultura }\end{array}$ \\
\hline & $\begin{array}{l}\text { 1.3. Escasez de } \\
\text { recursos }\end{array}$ & & $\begin{array}{l}3.3 . \\
\text { Desigualdad }\end{array}$ & & $\begin{array}{l}\text { 4.3. Poder } \\
\text { económico }\end{array}$ & & $\begin{array}{l}\text { 9.3 Distorsión } \\
\text { de la } \\
\text { información }\end{array}$ \\
\hline & $\begin{array}{l}\text { 1.4. Crisis } \\
\text { ambiental }\end{array}$ & & $\begin{array}{l}\text { 3.4. Falta de } \\
\text { seguridad }\end{array}$ & \multirow[b]{2}{*}{ 5. Educación } & $\begin{array}{l}\text { 5.1. Falta de } \\
\text { educación }\end{array}$ & & 9.4. Drogas \\
\hline & $\begin{array}{l}\text { 1.5. Medio } \\
\text { Ambiente }\end{array}$ & & $\begin{array}{l}\text { 3.5. Falta de } \\
\text { trabajo }\end{array}$ & & $\begin{array}{l}\text { 5.2. Problemas } \\
\text { en la } \\
\text { educación }\end{array}$ & & 9.5 Explotación \\
\hline & $\begin{array}{l}1.6 . \\
\text { Sobrepoblación }\end{array}$ & & 3.6. Ilegalidad & \multirow{2}{*}{ 6. Salud } & $\begin{array}{l}\text { 7.1. Falta de } \\
\text { Salud }\end{array}$ & & 9.6. Inseguridad \\
\hline \multirow{5}{*}{ 2. Conflictos } & 2.1. Personales & & $\begin{array}{l}\text { 3.7. Injusticia } \\
\text { Social }\end{array}$ & & $\begin{array}{l}\text { 7.2. Sistema de } \\
\text { salud }\end{array}$ & & $\begin{array}{l}\text { 9.7. Mal uso de } \\
\text { la tecnología }\end{array}$ \\
\hline & 2.2. Violencia & & $\begin{array}{l}\text { 3.8. crisis } \\
\text { económica }\end{array}$ & 7.Globalización & & & \multirow{2}{*}{$\begin{array}{l}\text { No válidas/no } \\
\text { categorizados }\end{array}$} \\
\hline & $\begin{array}{l}\text { 2.3. Ausencia } \\
\text { de paz }\end{array}$ & & $\begin{array}{l}3.9 . \\
\text { Discriminación }\end{array}$ & 8. Política & & & \\
\hline & \multirow[t]{2}{*}{ 2.4. Conflictos } & & $\begin{array}{l}3.10 \\
\text { Problemas de } \\
\text { la infancia }\end{array}$ & & & & \\
\hline & & & 3.11 Migración & & & & \\
\hline
\end{tabular}


Como resultado hemos obtenido un total de 857 problemas organizados mediante el sistema de categorías y subcategorías. Hemos optado por analizar las variables ambiental y social en dos categorías separadas para facilitar el análisis teniendo en cuenta en la elaboración de conclusiones el enfoque holístico y pluridimensional desde el que nos situamos sobre la problemática socioambiental (Azcárate, Navarrete y García, 2012). En la Tabla 2 se presenta el resultado de los problemas organizados en categorías y sus frecuencias. Problemas sociales, ambientales y conflictos son las tres categorías con mayor frecuencia en este orden.

Tabla 2: Problemas organizados por categorías. Frecuencias y porcentajes

\begin{tabular}{|c|l|c|c|}
\hline № & Categoría & Frecuencia & $\%$ \\
\hline 3 & Problemas Sociales & 271 & 31,6 \\
\hline 1 & Problemas Ambientales & 239 & 27,9 \\
\hline 2 & Conflictos & 168 & 19,6 \\
\hline 4 & Poder & 50 & 5,8 \\
\hline 5 & Educación & 38 & 4,4 \\
\hline 6 & Salud & 12 & 1,4 \\
\hline 8 & Política & 11 & 1,3 \\
\hline 7 & Globalización & 4 & 0,5 \\
\hline 9 & Otras & 23 & 2,7 \\
\hline 0 & Sin categoría & 41 & 4,8 \\
\hline
\end{tabular}

En la Figura 1 se presenta el análisis de los problemas por subcategorías. Para la interpretación de los datos hemos considerado significativo aquellos problemas que son mencionados al menos por el $15 \%$ de los encuestados. En un contexto social de posconflicto se observa cómo la violencia sigue siendo el problema de mayor preocupación para los maestros en formación encontrándose en conexión por su relevancia e importancia con la contaminación, la pobreza, la mala situación del medio ambiente, la escasez de recursos, la crisis ambiental y el cambio climático. Los problemas detectados en la educación revelan la preocupación por la existencia de una crisis de valores desencadenante y en conexión con los conflictos socioambientales. Estas conexiones claves entre violencia, problemas ambientales y crisis de valores y de educación deben ser explicadas y entendidas en las propuestas de formación de educación ambiental para la paz, de forma que se aborden las interconexiones entre las problemáticas y de qué manera unas son consecuencias de otras con el propósito de comprender de forma compleja y sistémica sus causas y consecuencias.

\section{Problemas del mundo}

1.1. Cambio climático

1.2. Contaminación ambiental

1.3. Escasez de recursos

1.4. Crisis ambiental

1.5. Medio Ambiente

2.2. Violencia

3.1. Crisis de valores

3.2. Pobreza

3.3. Desigualdad

4.2. Corrupción

5.2. Problemas en la educación

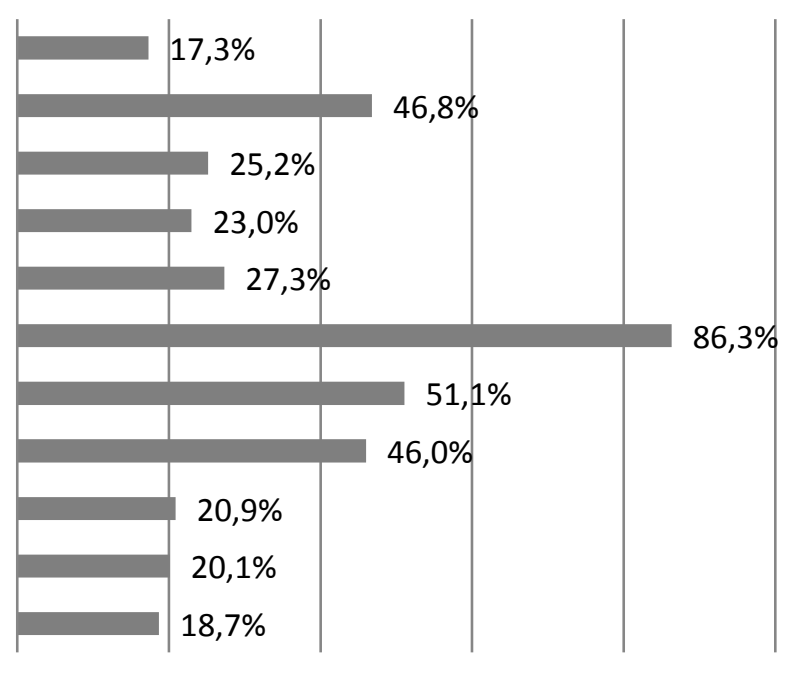

Fig. 1: Problemas del mundo por subcategorías 
A continuación presentamos resultados que responden al análisis del orden que los docentes atribuyen a los problemas en función del nivel de importancia para ellos (siendo el nivel 1 mayor importancia y nivel 5 menor importancia). En la Figura 2 se presenta los resultados organizados por categorías y subcategorías. De los resultados de niveles de importancia por categorías observamos que en el nivel 1 la categoría de problemas ambientales aparece un $40 \%$ seguida de la categoría conflictos con un $27,4 \%$ y categoría problemas sociales con un $21,6 \%$. El análisis por subcategorías nos revela que el problema de la violencia es el más mencionado con un $14,3 \%$ seguido de la contaminación ambiental con un $11,4 \%$ y el medio ambiente en general con un $6,7 \%$.

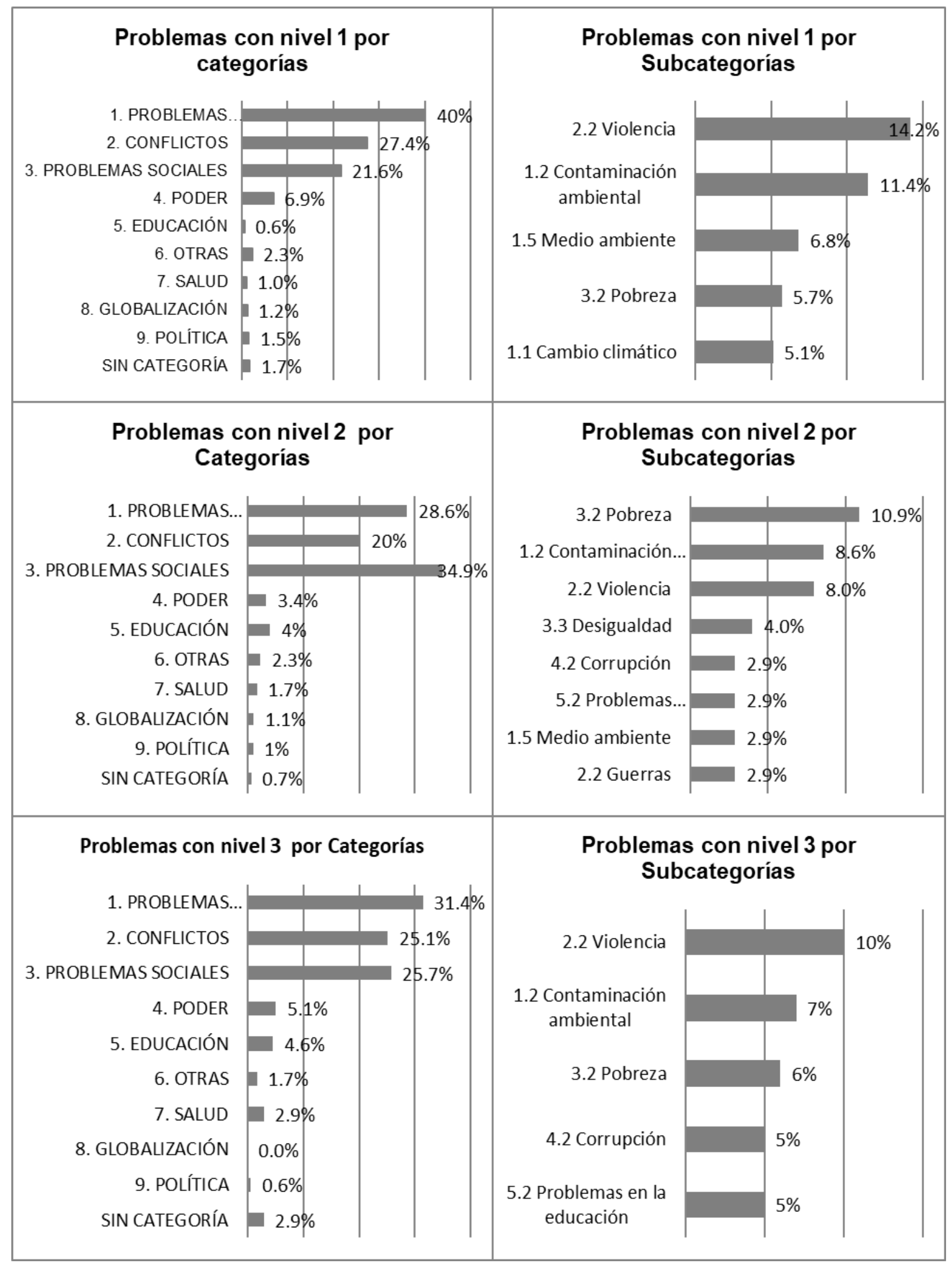

Fig. 2: Nivel de importancia de los problemas organizados por categorías y subcategorías 


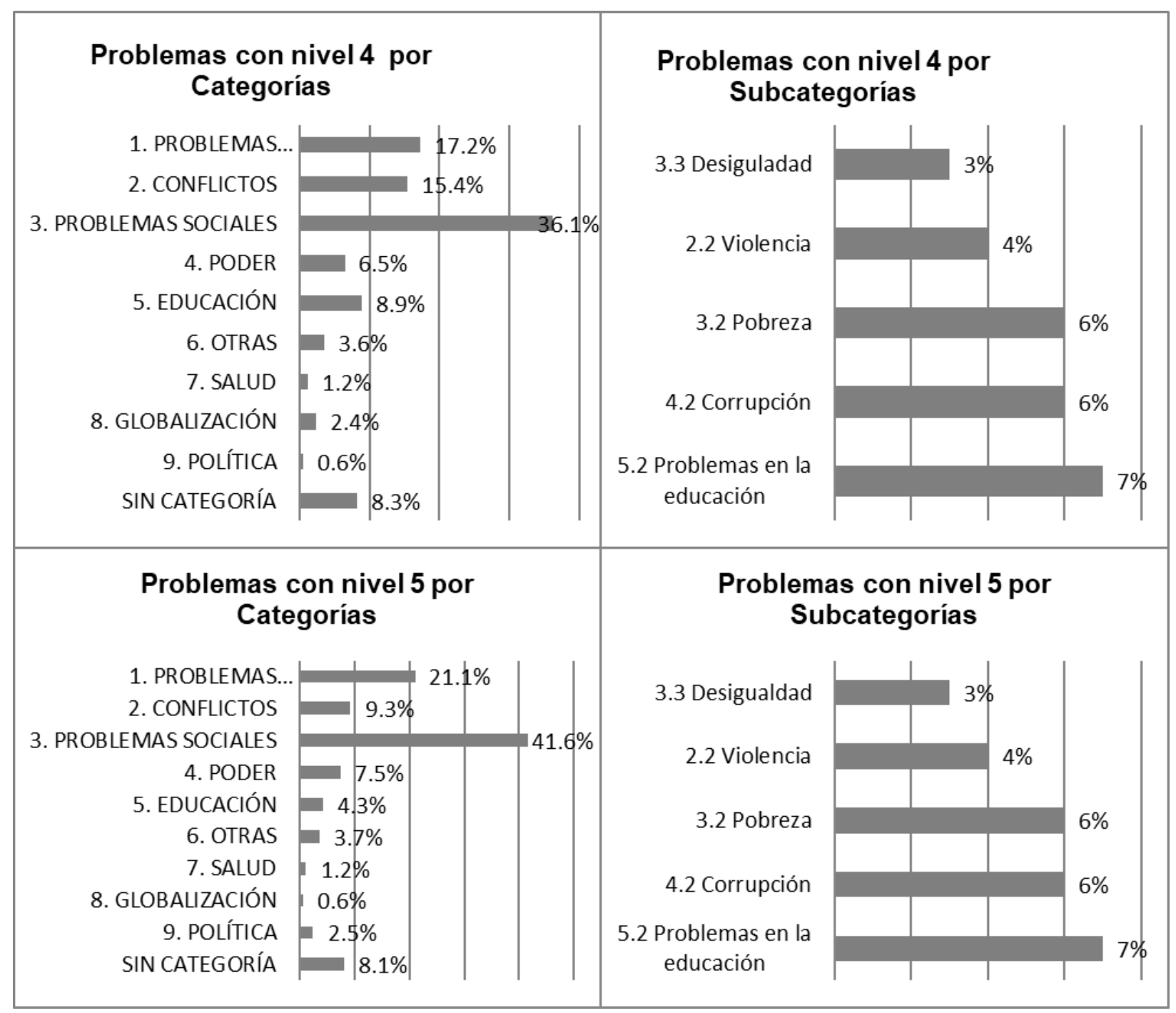

Fig. 2 (Continuación) : Nivel de importancia de los problemas organizados por categorías y subcategorías

En la gráfica de nivel 2 destaca la categoría de problemas sociales en primer lugar 34,9\% seguida de la categoría de problemas ambientales $28,6 \%$ y la categoría conflictos con un $20 \%$ del total de problemas indicados. La pobreza, la contaminación ambiental y la violencia son los problemas con mayor frecuencia de repetición. En la gráfica de nivel 3 se encuentra con mayor frecuencia la categoría problemas ambientales $(31,4 \%)$ seguida de la categoría conflictos y problemas sociales con un $25 \%$ cada una. En este nivel el problema de la violencia es el de mayor frecuencia seguida de la contaminación ambiental y la pobreza.

En la gráfica de nivel 4 la categoría problemas sociales se sitúa en primer lugar con un $36,1 \%$ seguida de la categoría problemas ambientales con un 17,2\% y categoría conflictos con un 15,4\%, aparecen otras categorías, pero con menor frecuencia. En este nivel aparece la subcategoría problemas en la educación como la de mayor frecuencia seguida de corrupción y pobreza. En el nivel 5 de importancia se sitúa la categoría problemas sociales como la de mayor frecuencia con un $41,6 \%$ seguida de la categoría problemas ambientales $21,1 \%$ y la categoría conflictos $9,3 \%$. Pobreza, corrupción y contaminación ambiental son los problemas con mayor frecuencia a este nivel. Como resultado general observamos que existe una interconexión directa a nivel de preocupación e importancia entre el trinomio violencia-contaminación-pobreza. Es fundamental que una formación en educación ambiental para la paz recoja las inquietudes y preocupaciones sociales desde un enfoque crítico, en este caso para analizar cómo son las relaciones que se establecen en este trinomio.

En la Figura 3 presentamos la evolución que experimentan las categorías según el nivel de importancia. En coherencia con los problemas de mayor frecuencia, las categorías ambiental, conflicto y social son las más relevantes en el análisis transversal por niveles de importancia. Esto refuerza la necesidad de incorporar estas preocupaciones socioambientales en el desarrollo de estrategias pedagógicas para la paz y la sostenibilidad. 


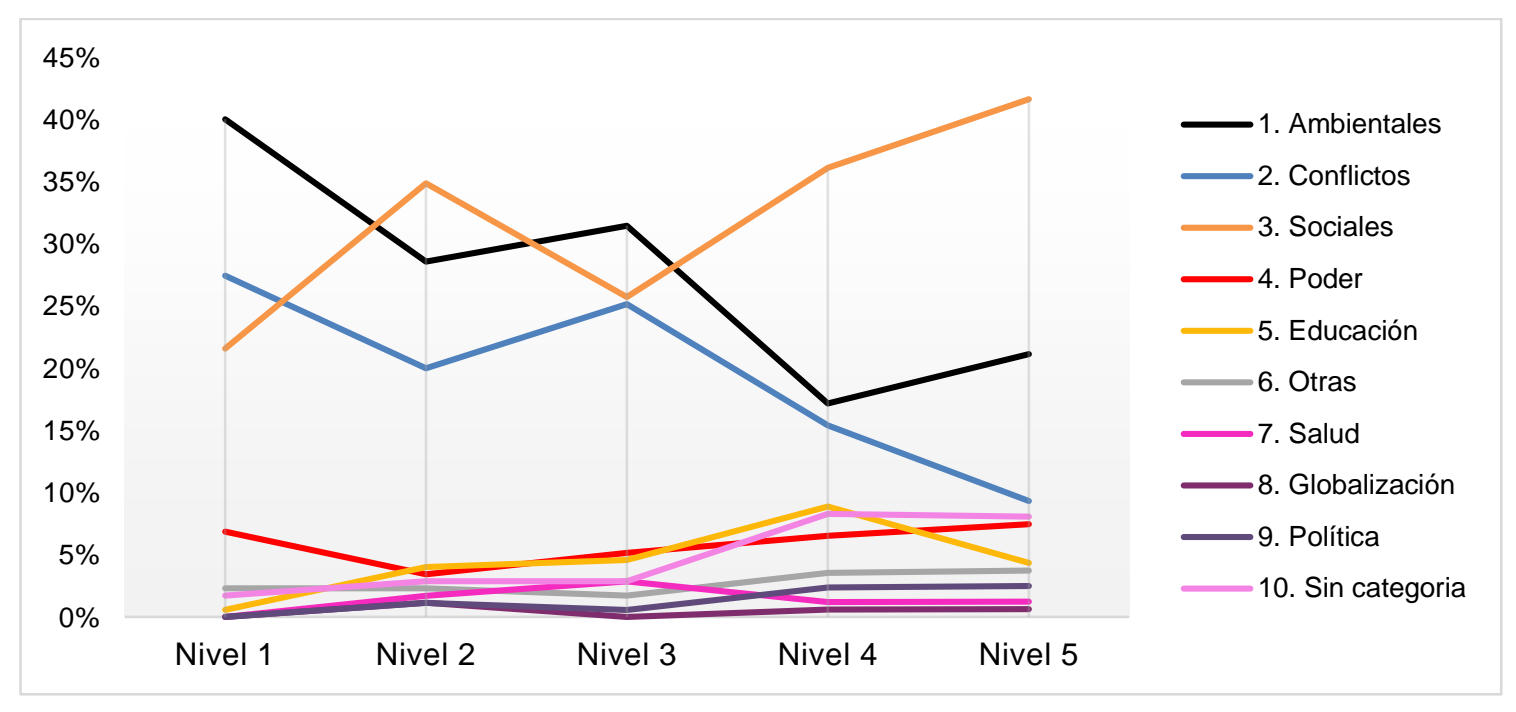

Fig. 3: Evolución de las categorías por niveles

\section{Pregunta 2:}

¿Qué crees que puedes aportar para la resolución de estos problemas? La Tabla 3 muestra las propuestas de los participantes organizadas en categorías emergentes a partir de los datos: De un total de 206 propuestas, se observa que los estudiantes principalmente realizan aportaciones de carácter educativo como contribuciones para la resolución de las problemáticas planteadas. En esta categoría destacan propuestas relacionadas con la educación ambiental y la educación en valores. En la categoría personal destacan propuestas relacionadas con el compromiso y responsabilidad individual para ser ejemplo como docente. En la categoría pensamiento crítico aluden a la formación para el desarrollo de la conciencia sobre las problemáticas.

Tabla 3: Categorías de análisis.

\begin{tabular}{|c|c|c|}
\hline Educación & $\begin{array}{l}\text { Son propuestas en las que mencionan la educación como principal herramienta } \\
\text { para la resolución de los problemas. Dentro de esta categoría hemos agrupado las } \\
\text { respuestas en tres subcategorías: educación ambiental, educación en valores y } \\
\text { educación en general por hacer mención concreta y ser bastante coincidente entre } \\
\text { los encuestados }\end{array}$ & $53 \%$ \\
\hline Personal & $\begin{array}{l}\text { Son propuestas que parten del compromiso individual como persona en la } \\
\text { coherencia interna, siendo responsable y ejemplo como educador. }\end{array}$ & $20 \%$ \\
\hline $\begin{array}{l}\text { Pensamiento } \\
\text { crítico }\end{array}$ & $\begin{array}{l}\text { Son propuestas que hacen un alegato a la reflexión personal desde una } \\
\text { conciencia y pensamiento crítico. }\end{array}$ & $12 \%$ \\
\hline \multirow[t]{2}{*}{ Otros } & Son propuestas diversas con escasa frecuencia de repetición. & $6 \%$ \\
\hline & No categorizable & $3 \%$ \\
\hline
\end{tabular}

\section{Pregunta 3:}

Escribe cinco palabras claves que te sugiera el concepto de Sostenibilidad. Para el análisis de la visión que tienen los estudiantes de la Maestría en Pedagogía sobre sostenibilidad hemos utilizado el programa de análisis cualitativo Atlas.ti. Los resultados nos ofrecen un total de 482 palabras agrupadas por familias de palabras (Tabla 4). En la siguiente Figura 5 se muestra los principales resultados a partir de sus frecuencias mediante una nube de palabras. En cuanto a las palabras claves que les sugiere el concepto de sostenibilidad se aprecia cierta relevancia de elementos pertenecientes tanto a aspectos físicos (ambiente, recursos), socioculturales (conciencia, responsabilidad, cuidado, equilibrio, educación) cómo económicos (desarrollo, economía, consumo, producción y trabajo) haciéndose eco del concepto difundido por el Informe Brundtland (1987) de gran calado internacional. Sin embargo se detectan ausencias fundamentales para comprender la problemática ambiental desde un enfoque holístico y que están presente en los Objetivos de Desarrollo Sostenible (tales como fin de la pobreza, igualdad de género, hambre cero, salud y bienestar, paz, ecojusticia y reducción de desigualdades). Por otro lado se detecta la ausencia de componentes claves que forman parte de los actuales enfoques metodológicos 
de la educación ambiental basados en visiones sistémicas y complejas que fomenten el pensamiento ético crítico para una participación política y social activa; componentes esenciales de la sostenibilidad.

Es de destacar el término Conciencia como el elemento con mayor frecuencia de identificación con el concepto de sostenibilidad. Esto puede deberse al protagonismo que actualmente se le asigna a la conciencia ambiental en los planes y programas de educación ambiental. Como ejemplo, los estudios de Mora-Ortiz (2015) tras una revisión de los PRAE destacan cómo el fomento de la conciencia ambiental es el tema más referenciado entre los objetivos y propósitos de los documentos. Del mismo modo en los estudios de Muñoz y Caicedo (2017) se consideran los PRAE como un eje primordial de la actividad escolar para construir conciencia ambiental para el cambio de actitudes.

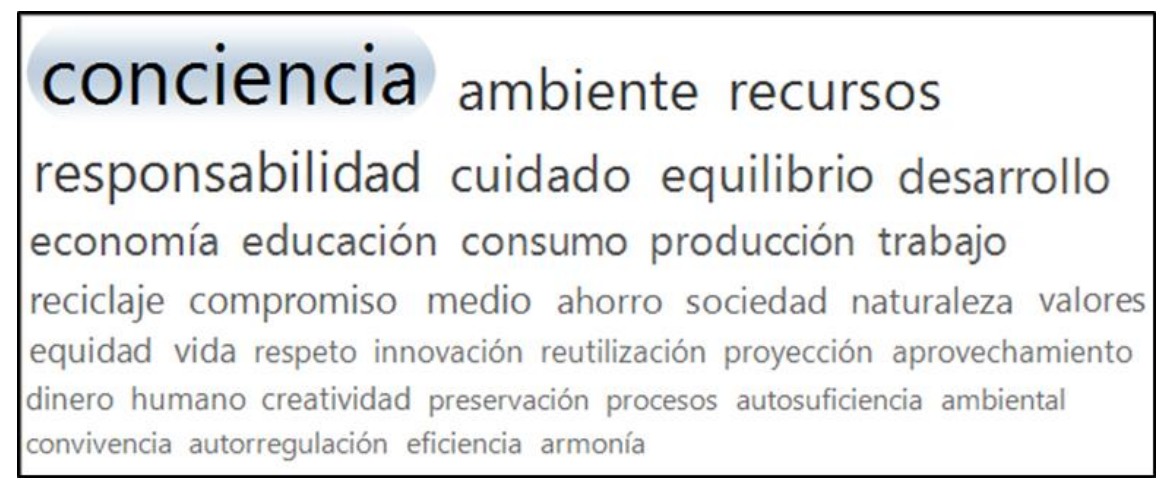

Fig. 4: Nube de palabras sobre el concepto de sostenibilidad.

Tabla 4: Frecuencia de repetición de palabras que definen sostenibilidad.

\begin{tabular}{|l|l|l|l|l|l|}
\hline Conciencia & 25 & Cuidado & 13 & Educación & 9 \\
\hline Ambiente & 19 & Equilibrio & 12 & Consumo & 9 \\
\hline Recursos & 17 & Desarrollo & 11 & Producción & 10 \\
\hline Responsabilidad & 15 & Economía & 10 & Trabajo & 8 \\
\hline
\end{tabular}

\section{Segunda parte del cuestionario.}

Este apartado recoge los datos del cuestionario tipo Likert. Los maestros deben responder según el grado de acuerdo con las afirmaciones, siendo el 1 el valor mínimo o de completo desacuerdo, 2 desacuerdo, 3 tendente al acuerdo, 4 de acuerdo, 5 muy de acuerdo. Las preguntas están organizadas atendiendo a tres dimensiones: A) visión sistémica, compleja y crítica de la problemática socioambiental; B) concepciones en cuanto a los fines de la educación ambiental en el contexto escolar y C) estrategias para ir del pensamiento a la acción. A continuación presentamos los resultados organizados por dimensiones:

\section{Dimensión A:}

Esta dimensión está compuesta por cuatro ítems relacionados con la visión de la problemática ambiental, la ideología del educador y los fines de la educación. En la Tabla 5 presentamos la frecuencia de respuesta en escala Likert de cada ítem. En el ítem 1 "la pobreza es un problema ambiental" se esperaban unos resultados en el que el nivel 5 tuviera mayor presencia. Sin embargo estos datos nos revelan la necesidad de profundizar en las interconexiones de las problemáticas anteriormente declaradas especialmente en el trinomio violenciacontaminación-pobreza.

Tabla 5: Datos dimensión A.

\begin{tabular}{|c|c|c|c|c|c|c|}
\hline Dimensión A / (Escala Likert 1-5) & 1 & 2 & 3 & 4 & 5 & Nulo \\
\hline 1. La pobreza es un problema ambiental & 29 & 26 & 26 & 36 & 51 & 7 \\
\hline $\begin{array}{l}\text { 2. Un docente debe presentar los contenidos evitando que reflejen su } \\
\text { propia ideología. }\end{array}$ & 51 & 35 & 32 & 30 & 22 & 5 \\
\hline 3. Hay que concienciar al alumnado, pero sin meternos en política. & 41 & 22 & 31 & 45 & 34 & 2 \\
\hline $\begin{array}{l}\text { 4. Hay que potenciar estrategias de resolución de conflictos para una } \\
\text { transformación social. }\end{array}$ & 5 & 1 & 2 & 15 & 147 & 5 \\
\hline
\end{tabular}


Respecto al ítem 2 "Un docente debe presentar los contenidos evitando que reflejen su propia ideología" la tendencia es mayor en el nivel poco de acuerdo aunque tampoco hay grandes diferencias. Desde una educación para la paz se espera que el docente sea consciente de su propia ideología y de la influencia que tiene sobre el alumnado y de la importancia de ser coherente entre lo que se quiere enseñar y la conducta personal. Estas respuestas son acordes con los resultados obtenidos en la pregunta 2 sobre las aportaciones para resolver problemas, ya que existe una amplia mayoría que plantean la vía de la educación y de ser ejemplos como educadores.

En relación al ítem 3 "Hay que concienciar al alumnado, pero sin meternos en política" el grado de acuerdo está distribuido casi proporcionalmente entre los 5 niveles. Este resultado revela la necesidad que desde la educación ambiental para la paz se profundice en el concepto de política, de democracia ambiental y de cómo hacer política para la sostenibilidad. En el ítem 4 "Hay que potenciar estrategias de resolución de conflictos para una transformación social" existe un gran acuerdo en la mayoría de los participantes sobre la necesidad de potenciar herramientas para la resolución de conflictos. Resultado coherente con los datos de la primera parte del cuestionario sobre la violencia como uno de los problemas de mayor preocupación en los participantes.

Como resultado global de esta categoría, uno de los propósitos para desarrollar en la formación en educación ambiental para la paz debe centrarse en la profundización de las conexiones existentes entre causas, consecuencias y enfoque político de la problemática socioambiental de manera particular pero con una visión sistémica global como plantean los estudios (Bonil, Junyent y Pujol, 2010; Murga-Menoyo y Novo, 2017).

\section{Dimensión B:}

concepciones en cuanto a los fines de la educación ambiental en el contexto escolar, formada por tres ítems relacionados con diferentes enfoques de educación ambiental. En la Tabla 6 presentamos la frecuencia de respuesta en escala Likert de cada ítem. Esta dimensión pretende investigar sobre cómo entienden los participantes los fines de la educación ambiental en el contexto escolar con el propósito de realizar una aproximación sobre el sentido que para los docentes tiene para ellos la educación ambiental. De los datos de la Tabla 6 subrayamos que en el ítem 5 "Conocer la Naturaleza y aprender a disfrutar en ella, a amarla y a conservarla" destaca de forma considerable el nivel 5 (muy de acuerdo). Esta afirmación se relaciona con modelos de enseñanza en el que la educación ambiental se identifica con el desarrollo de valores como el amor o la empatía para la conservación. Atendiendo a los estudios de García-Díaz (2004) este enfoque como fin educativo es necesario pero se queda incompleto en relación a otros enfoques más orientados a la al desarrollo de estrategias encaminadas a la acción y que realmente aborden los problemas socioambientales y provoquen cambios de mayor calado en el alumnado.

En relación con el ítem 6 "Facilitar el desarrollo de destrezas y de hábitos proambientales" observamos que la mayoría se sitúa en el nivel 5 (muy de acuerdo), un enfoque más centrado en el activismo, enfoque predominante entre los educadores ambientales como proponen las investigaciones de Rodríguez Marín y García-Díaz (2009), sin embargo en el ítem 7 la mayoría está de acuerdo con que la acción también vaya acompañada de pensamiento para el cambio. Esta última finalidad estaría más acorde con una educación ambiental para la paz enfocada hacia una transformación personal para la transformación del sistema.

Tabla 6: Datos dimensión B.

\begin{tabular}{|l|c|c|c|c|c|c|}
\hline Dimensión B / (Escala Likert 1-5) & 1 & 2 & 3 & 4 & 5 & Nulo \\
\hline $\begin{array}{l}\text { 5. Conocer la Naturaleza y aprender a disfrutar en ella, a amarla y } \\
\text { a conservarla. }\end{array}$ & 4 & 0 & 9 & 21 & 139 & 2 \\
\hline $\begin{array}{l}\text { 6. Facilitar el desarrollo de destrezas y de hábitos proambientales } \\
\text { como separar las basuras, respetar y cuidar los seres vivos, } \\
\text { reciclar papel, etc. }\end{array}$ & 3 & 2 & 17 & 30 & 121 & 2 \\
\hline $\begin{array}{l}\text { 7. Desarrollar en el alumnado un pensamiento y una actuación } \\
\text { conducentes a cambiar el mundo en la dirección de hacerlo más } \\
\text { sostenible. }\end{array}$ & 5 & 0 & 3 & 14 & 152 & 1 \\
\hline
\end{tabular}

Dimensión C: estrategias para ir del pensamiento a la acción armada por cinco ítems relacionados con la práctica de la educación ambiental. En la Tabla 7 presentamos la frecuencia de respuesta en escala Likert de cada ítem. Observamos que en el ítem 8 "Intentar persuadir y convencer al alumnado de que tienen que responder a la gravedad de la actual crisis ambiental" hay una tendencia a estar más de acuerdo con la persuasión y convicción sobre formas de actuación más sostenibles. Con respecto ítem 9 "Hacer muchas salidas al medio y talleres de reciclaje" existe una distribución no muy diferenciada entre los niveles 3,4 y 5 . Estos resultados tienen cierta coherencia con el ítem 6 de desarrollo de destrezas y hábitos, ya que es un modelo de educación ambiental que continúa siendo muy extendido. 
Tabla 7: Datos dimensión C.

\begin{tabular}{|c|c|c|c|c|c|c|}
\hline Dimensión C/ (Escala Likert 1-5) & 1 & 2 & 3 & 4 & 5 & Nulo \\
\hline $\begin{array}{l}\text { 8. Intentar persuadir y convencer al alumnado de que tienen que } \\
\text { responder a la gravedad de la actual crisis ambiental apoyando } \\
\text { determinadas formas de actuación. }\end{array}$ & 9 & 13 & 34 & 52 & 64 & 3 \\
\hline 9. Hacer muchas salidas al medio y talleres de reciclaje. & 3 & 28 & 46 & 61 & 34 & 3 \\
\hline $\begin{array}{l}\text { 10. Procurar que expliciten y contrasten sus ideas en todo momento, } \\
\text { para que tomen conciencia de lo que piensan y para que movilicen } \\
\text { sus concepciones en la construcción de nuevos conocimientos. }\end{array}$ & 1 & 5 & 8 & 39 & 118 & 4 \\
\hline $\begin{array}{l}\text { 11. Ayudar a construir un pensamiento ético crítico partiendo del } \\
\text { análisis de la realidad. }\end{array}$ & 4 & 0 & 1 & 19 & 149 & 2 \\
\hline $\begin{array}{l}\text { 12. Desarrollar un pensamiento complejo que conlleve creatividad } \\
\text { social y ética civil. }\end{array}$ & 3 & 3 & 12 & 30 & 123 & 4 \\
\hline
\end{tabular}

Gran parte de los participantes están de acuerdo con el pensamiento de que las ideas de los alumnos son importantes para incluir contenidos de educación ambiental en sus clases de forma que ayuden a construir un pensamiento complejo a partir de la movilización de sus propias ideas. En la línea de los trabajos de Rengifo, Quitiaquez y Mora (2012), Straková y Cimermanová (2018) y Van Poeck y Östman (2018), nos ofrecen ciertas orientaciones sobre las concepciones que tienen los maestros sobre cuáles son las estrategias más adecuadas para incorporar la sostenibilidad en las aulas. De forma general se caracteriza por una enseñanza hacia un cambio cualitativo de valores y acciones, basados en un pensamiento ético y crítico, propuestas que siguen los lineamientos comunes de la educación ambiental y la educación para la paz.

\section{DISCUSION FINAL}

En relación al objetivo 1, en un contexto social de posconflicto se observa que el problema de la violencia cobra especial preocupación entre los participantes encontrando conexiones por relevancia con la contaminación y la pobreza.

En cuanto al objetivo 2 los participantes proponen la educación ambiental como alternativa para la resolución de los problemas socioambientales. Pero que para que sea eficaz es imprescindible que un docente sea coherente con sus pensamientos y actitudes, que se deben apoyar en el desarrollo de un pensamiento ético y crítico junto con la una acción para el cambio.

En relación al objetivo 3 se percibe en los participantes la influencia ejercida a lo largo de estos años del concepto de sostenibilidad infundido por el Informe Brundtland (CMMAD, 1988) detectando algunas ausencias de enfoques propuestos por los ODS y por las metodologías activas de la educación ambiental. Aparece reforzado el binomio sostenibilidad-conciencia fruto de su reforzamiento en los planes y programas educativos como los PRAE.

Atendiendo al objetivo 4 los participantes destacan de manera significativa un modelo de educación ambiental que fomente estrategias de resolución de conflictos en el aula para la transformación socioambiental. Entienden que entre los fines de la EA debe estar el conocimiento de la naturaleza desde una implicación emocional de cuidado y protección, pero que lleve a la acción para el cambio acompañado de un pensamiento crítico.

\section{CONCLUSIONES}

Atendiendo al fin último de este trabajo para una mejora en la formación en educación ambiental para la paz de los estudiantes de la Maestría en Pedagogía se concluye con las siguientes propuestas de mejora:

a) la incorporación de inquietudes y preocupaciones sociales de los estudiantes y del contexto social a los contenidos del programa, en concreto sobre el trinomio violencia-contaminación-pobreza y su tratamiento desde un enfoque crítico;

b) el abordaje de las conexiones entre violencia, problemas ambientales y crisis de valores y de educación de forma compleja y sistémica analizando sus causas y consecuencias;

c) la inclusión de enfoques y propuestas claves que forman parte de los actuales posicionamientos metodológicos de la educación ambiental basados en una educación ética crítica para una participación política y social activa para la paz y la resolución de conflictos; componentes esenciales de la sostenibilidad.

d) por último es importante reorientar estas propuestas formativas en coherencia con las finalidades de la Cátedra para la Paz y los PRAE, de forma que se fomenten las interconexiones, complementariedades y sinergias para una sociedad sostenible y no violenta. 


\section{REFERENCIAS}

Azcárate, P., Navarrete, A. y García, E., Aproximación al nivel de inclusión de la sostenibilidad en los curricula universitarios. Profesorado. Revista de currículum y formación de profesorado, 16(2), 105-119 (2012).

Bonil, J., M. J., y Pujol, R. M., Educación para la Sostenibilidad desde la perspectiva de la complejidad, Revista Eureka sobre Enseñanza y Divulgación de las Ciencias, 7, 198-215 (2010).

Brantmeier, E. J., Toward a critical peace education for sustainability. Journal of peace education, 10(3), $242-258$ (2013).

Cebrián, G. y Junyent, M., Competencies in Education for Sustainable Development: Exploring the Student Teachers' Views, Sustainability, 7, 2768-2786 (2015).

Cortés, A. y González. L., Dimensión Ambiental en el currículo de educación básica y media, Educación y Educadores, 20 (2), 382 - 399 (2017).

Decreto 1038 Por el cual se reglamenta la Cátedra de la Paz, Ministerio de Educación Nacional, Colombia, (2015).

Duarte, J. y Valbuena, E., Referentes de la formación de profesores en educación ambiental, Revisión de antecedentes 2000-2012, Uni-pluriversidad, 14(2), 27-36 (2014).

Espejel, A., y Flores, A., Educación ambiental escolar y comunitaria en el nivel medio superior, Puebla-Tlaxcala, México. Revista mexicana de investigación educativa, 17(55), 1173-1199 (2012).

García, J. E., Los contenidos de la Educación Ambiental: una reflexión desde la perspectiva de la complejidad, Revista Investigación en la Escuela, 53, 31-51 (2004).

Håkansson, M., Östman, L., y Van Poeck, K., The political tendency in environmental and sustainability education, European Educational Research Journal, 17(1), 91-111 (2018).

Kalsoom, Q., Khanam, A., y Quraish,i U., Sustainability consciousness of pre-service teachers in Pakistan. International Journal of Sustainability in Higher Education, 18(7), 1090-1107 (2017).

Lara, M., Informe sobre los proyectos de educación ambiental no formal, Revista Iberoamericana de Educación, 11, 153169 (1996).

Ley 1732 Por la cual se establece la Cátedra de la Paz en todas las Instituciones educativas del país, Congreso de Colombia, (2014).

Mora-Ortiz, J. R. Los Proyectos Ambientales Escolares. Herramientas de gestión ambiental. Revista Bitácora Urbano Territorial, 25(2), 67-74 (2015).

Muñoz, C. R. R. y Caicedo, A. E. G., Una mirada desde la universidad a la educación ambiental en el nivel básico primaria de Colombia. Revista Conrado, 13(1), 37- 43 (2017).

Murga-Menoyo, M., y Novo, M., Sostenibilidad, desarrollo “glocal» y ciudadanía planetaria. Referentes de una Pedagogía para el desarrollo sostenible, DOI: http://dx.doi.org/10.14201/teoredu20172915578, Teoría de la Educación, 29(1), 55-78 (2017)

Nussbaum, M. C., Patriotism and cosmopolitanism i: Cohen, For Love of Country: Debating the Limits of Patriotism, Martha C Nussbaum with respondents (1996).

Oviedo, H., y Campos-Arias, A., Aproximación al uso del coeficiente Alfa de Conbrach. Revista Colombiana de Psiquiatría, XXXIV (4), 572-580 (2005).

Reardon, B., Peace Education: A Review and Projections, Vol.17, Malmo: Malmo University School of Education, (1999).

Rengifo, B., Quitiaquez, L., y Mora, F., La educación ambiental una estrategia pedagógica que contribuye a la solución de la problemática ambiental en Colombia, XII Coloquio internacional de Geocrítica. Colombia, Recuperado de: http://www. ub. edu/geocrit/coloquio2012/actas/06-B-Rengifo.pdf. (2012).

Rieckmann, M., Education for sustainable development goals: Learning objectives, Publishing UNESCO, (2017).

Rodríguez Marín, F. y García Díaz, J. E., El activismo que no cesa. Obstáculos para incorporar la metodología didáctica basada en la investigación del alumno a la práctica de la Educación Ambiental. Revista Investigación en la Escuela, 67 , 23-36 (2009).

Rodríguez, J. J. F., ODS 16: paz, justicia e instituciones fuertes, bie3: Boletín IEEE, 11, 970-1001 (2018).

Straková, Z. y Cimermanová, I., Critical thinking development-A necessary step in higher education transformation towards Sustainability, Sustainability, 10(10), 3366 (2018).

Shapiro, S., Globalization and Pedagogy of Peace, In Second International Handbook on Globalisation, Education and Policy Research, Springer, Dordrecht, 577-588 (2015).

Solís-Espallargas, C., y Valderrama-Hernández, R., La educación para la sostenibilidad en la formación de profesorado ¿Qué estamos haciendo?, Foro de Educación, 13(19), 165-192 (2015).

Spahiu, M., y Lindemann-Matthies, P., Effect of a Toolkit and a One-Day Teacher Education Workshop on ESD Teaching Content and Methods-A Study from Kosovo, Sustainability, 7(7), 8051-8066 (2015). 
Swee-Hin, T. y Cawagas, V. F., Peace education, ESD and the earth charter: Interconnections and synergies, Journal of Education for Sustainable Development, 4(2), 167-180 (2010).

Tal, T. y Peled, E., The philosophies, contents and pedagogies of environmental education programs in 10 Israeli elementary schools, Environmental Education Research, 23(7), 1032-1053 (2017).

United Nations. Transforming our world: The 2030 agenda for sustainable development; United Nations: New York, EE. U.U., (2015).

Varela-Losada, M., Pérez-Rodríguez, U., Álvarez-Lires, F. J. y Álvarez-Lires, M. M., Desarrollo de competencias docentes a partir de metodologías participativas aplicadas a la Educación Ambiental, Formación universitaria, 7(6), 27-36 (2014).

Verhagen, F. C., Sustainable communities: a lens for envisioning and achieving a community-based culture of social and ecological peace, Journal of Peace Education, 11(3), 297-316 (2014).

World Bank; Human Security Report Project, Miniatlas of Human Security. Brighton, UK: Myriad Editions, (2008). 
